\section{Feasibility study on retinal vascular bypass surgery in isolated arterially perfused caprine eye model}

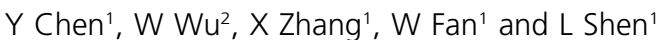

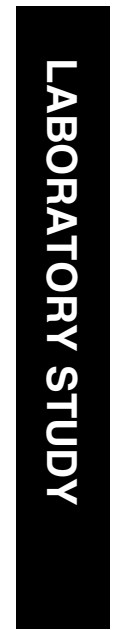

Eye (2011) 25, 1499-1503; doi:10.1038/eye.2011.197; published online 16 September 2011

Keywords: retinal vein occlusion; retinal vein bypass surgery; isolated arterially perfused caprine eye; closed-sky eyeball; keratoprosthesis

\section{Introduction}

As a very common retinal vascular disorder, probably second only to diabetic retinopathy, ${ }^{1}$ branch retinal vein occlusion (BRVO) is short of effective therapies at present. Recent research of this subject has mostly focused on the re-establishment of retinal venous outflow, including intravascular injection of vasoactive substances, ${ }^{2}$ arteriovenous crossing sheathotomy, ${ }^{3-6}$ and chorioretinal anastomosis. ${ }^{7-9}$ Bypassing the occluded retinal vessel segment with artificial graft, similar to clinical cure for myocardial ischemia using coronary artery bypass grafting, has been demonstrated feasible in an animal eyecup model. ${ }^{10}$ However, it did not represent the feasibility of retinal bypass surgery in the living eye as open-sky approach used in the above study was far from the real condition.

To further demonstrate the feasibility of retinal vein bypass surgery in this study, we performed micro-vascular maneuvers in a closed, arterially perfused caprine eyeball using temporary keratoprosthesis (TKP) vitrectomy approach.

Materials and methods

Preparation of isolated eye model

A total of 38 fresh caprine eyes, each with $\sim 1.0-1.5 \mathrm{~cm}$ of attached optic nerves, were
${ }^{1}$ Eye Hospital of Wenzhou Medical College, Wen Zhou, China

${ }^{2}$ The Second Affiliated Hospital of Wenzhou Medical College, Wen Zhou, China

Correspondence: L Shen, Department of Vitreoretinal Surgery, Eye Hospital of Wenzhou Medical College, 270 Xue-Yuan West Road, Wen Zhou, Zhe Jiang 325000, China Tel: + 86 57788068864; Fax: + 8657788832083 . E-mail: slj@mail.eye.ac.cn

Received: 15 June 2010 Accepted in revised form: 4 July 2011

Published online: 16 September 2011 
used in the study. The ophthalmic artery of each eye was separated from optic nerve adipose tissue and musculature. A 24-gauge intravenous indwelling needle, which was linked to an infusion bottle of Krebs solution via an infusion tube, was inserted into the broken arterial end. To avoid slippage, a 7-0 suture was used to ligate the ophthalmic artery and intravenous indwelling needle tightly. Krebs solution (osmotic to cell) was perfused into retinal vessels via the ophthalmic artery. The flow rate, which was controlled manually by adjusting the height of the flooding bottle, began at $0.2-0.4 \mathrm{ml} / \mathrm{min}$ and built gradually to $2.0-2.5 \mathrm{ml} / \mathrm{min}$. After complete displacement of the blood by Krebs solution, acid red G (100 mg/l) was injected into the retinal vessel from the infusion tube to stain the vessel red (Figure 1a).

\section{TKP vitrectomy}

The caprine stabilized on a Styrofoam mount under an operating microscope (Topcon OMS-800) (Topcon Corporation, Tokyo, Japan) was prepared for a standard three-port pars plana vitrectomy (PPV) using TKP. An infusion port was introduced into a sclerostomy at $3.0 \mathrm{~mm}$ limbal distance in the region of presumed pars plana. An $8.0-\mathrm{mm}$ vacuum trephin (Hessburg-Barron, JEDMED Instrument Company St Louis, MO, USA) was used for the central corneal trephination. An 8.25-mm Landers wide-field TKP (OLTK-8.2, Ocular Instruments Inc., Bellevue, WA, USA) was then inserted into the opening, and attached tightly to the corneoscleral limbus by 8-0 suture. Next, PPV and lensectomy were performed with the Vitrector (Storz Millenium FACO Y Vitrector, Bausch \& Lomb, Rochester, NY, USA). Triamcinolone was used to visualize the transparent vitreous cortex during vitreoretinal surgery, ${ }^{11}$ and complete removal of the posterior vitreous cortex was achieved. Corectomy was performed after lentectomy to attain a wide surgical field for retina micro-vascular maneuvers, because the small pupil of in vitro eye was not capable of being dilated by the drug.

\section{Establishment of BRVO model}

Experimental BRVO was induced by intraoperative endodiathermy of the main vein segment at a site just in the vessel's first branching (Figure 1b). Endodiathermy was applied with an endodiathermy unit (Storz Millenium FACO Y Vitrector, Bausch \& Lomb) in continuous mode with the power setting at 8-10. To confirm the complete occlusion of the treated vessel, sub-methyl-blue solution was injected into the retinal vessel via the ophthalmic artery (Figure 1c).

\section{Design of bypass vessel}

Polyimide tubes $(50.8 \mu \mathrm{m}$ internal diameter, $7.6 \mu \mathrm{m}$ wall thickness) (HV Technologies, Trenton, GA, USA) were used as artificial vessels. To match the angle between the main vein and the target branch vein straddling the occluded vein segment, the tubing was designed to a certain degree of curvature with the method of heat setting. Polyimide tube was then intercepted to the length of $6-8 \mathrm{~mm}$, which was $\sim 2 \mathrm{~mm}$ longer than the suppositional distance between the two vessel wall openings that would be created in the next stage, and the two ends were cut to $\sim 30^{\circ}$ slope. To replace the air in the tubing, Krebs solution was injected via borosilicate glass microtips ( $20 \mu \mathrm{m}$ external diameter, $20^{\circ}$ angled tip) (Eppendorf, Shanghai, China) into the polyimide tube.

\section{Retinal vein bypass}

All retinal micro-vascular manipulations were performed under keratoprosthesis.

\section{Vascular puncture}

A custom-made puncture needle with the tip of a sharp 12-0 circular suture needle jointing a flat headed 26-gauge syringe needles was used to pierce the vessel to create a small round hole at the most vitreal aspect of the vessel wall. Two such openings were created several millimeters apart at selected sites, one in the main vein wall and the other in one of its branch vein wall. Each opening was $\sim 2-3 \mathrm{~mm}$ away from the occlusion lesion. The ideal diameter of the opening was $\sim 60-70 \mathrm{~mm}$, which could allow access of a polyimide tubing end. The maneuver should be done gently to avoid over size aperture of opening and vascular laceration, which would cause failure of anastomosis in the next steps.

\section{Vascular catheterization}

Catheterization of the selected vein with a polyimide tube was carried out after vascular puncturing. A piece of prepared polyimide tubing was introduced into the vitreous cavity through sclerotomy. Both ends of the tubing were advanced into the vessel by a distance of $\sim 1 \mathrm{~mm}$, creating a vascular bypass between the two openings bridging the occlusion lesion.

\section{Sealed connection}

A sealed connection between the vessel and tubing was achieved by intraoperative endodiathermy of 

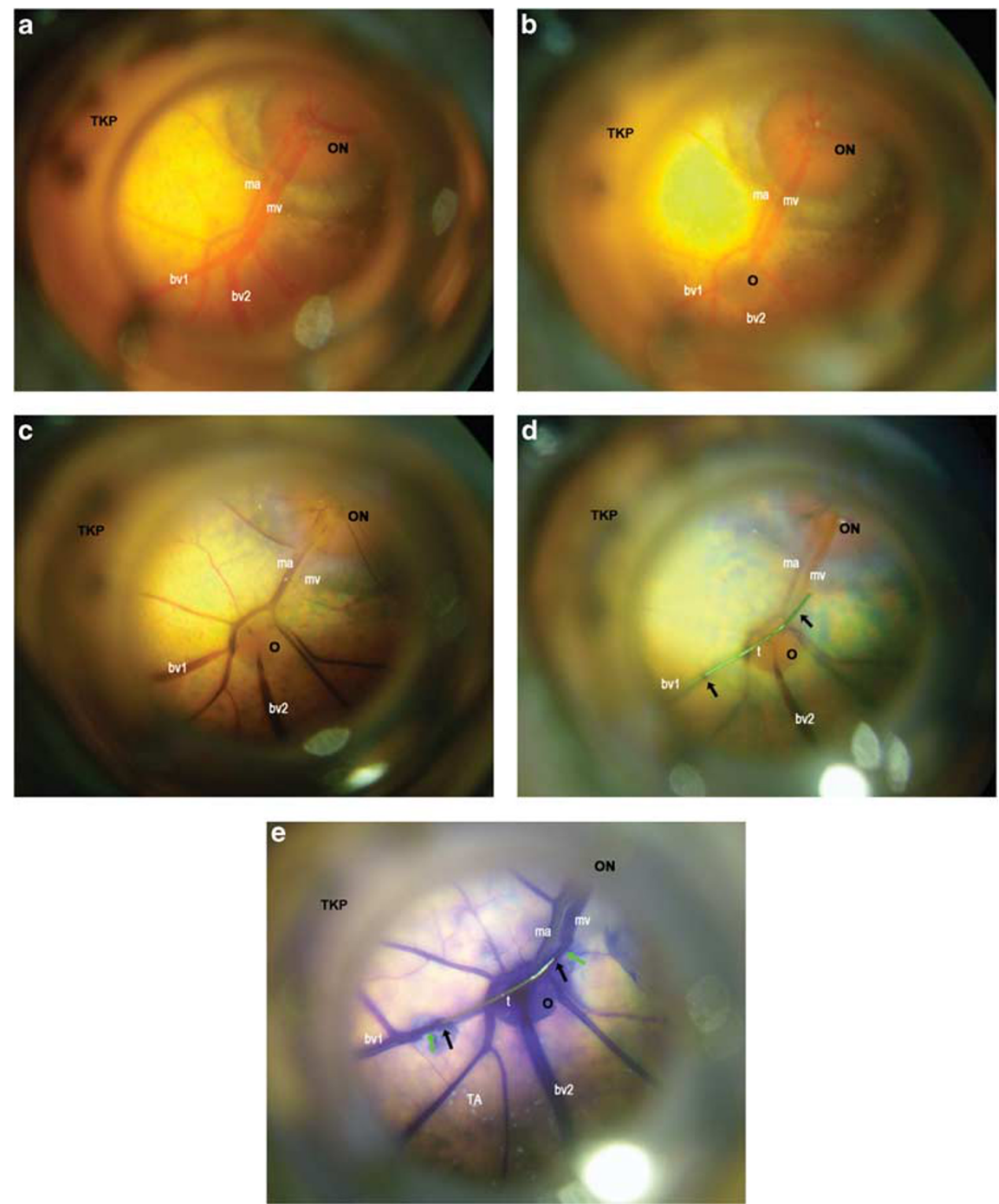

Figure 1 (a) Retinal vascular perfusion. Complete displacement of blood by Krebs solution made the retinal vessel appear transparent. Then, the retinal vessel appeared red after injection of acid red G. (b) Creation of BRVO model. Occlusion of a retinal main vein was established by vascular endodiathermy at a site just in the vessel's first branching. (c) Confirmation of vein occlusion. After sub-methyl-blue solution was injected into the retinal vessel, the distal branch vein stained blue and the main vein remained light red. (d) Retinal vein bypass surgery. Each end of a polyimide tube was inserted into separate vessel wall openings (black arrows), which had been created several millimeters apart at selected sites - one in the main vein wall and the other in one of its branch vein wall. Then, endodiathermy was performed to obtain a sealed connection between the vessel and the polyimide tube. (e) Patency of the retinal vascular bypass. After being injected into the retinal vessel, sub-methyl-blue solution flowed into the main vein vessel from the branch vessel through the vascular bypass, staining the main vein blue. Black arrows, vessel wall openings; green arrows, sealed connection between the vessel and polyimide tube by endodiathermy; bv, branch vein; ma, main artery; mv, main vein; $\mathrm{O}$, occluded side; $\mathrm{ON}$, optical nerve; $\mathrm{t}$, polyimide tube; TA, triamcinolone acetonide; TKP, temporary keratoprosthesis.

anastomosis with the power at setting 8-10 for several seconds.

Bypass of an occluded vein segment was achieved via the combination of vascular puncturing, probing, catheterization, and endodiathermy (Figure 1d).

\section{Evaluation of the patency of the retinal vascular bypass}

Patency of the vascular bypass was evaluated by examining the color of the retinal vessel after injection of sub-methyl-blue $(1 \mathrm{mg} / \mathrm{ml})$ into the retinal vessel through ophthalmic artery (Figure 1e). 


\section{Results}

\section{Establishment of BRVO model}

The treated vein was completely blocked as judged by the sub-methyl-blue method in 34 caprine eyes, with evident minimal macroscopic tissue damages at the selected sites (Figure 1b). In these eyes, the branch vein vessel distal to the occlusive segment was distended and blue, and the main vein was still light red (Figure 1c).

\section{Creation of the retinal vein bypass}

Puncturing the retinal vessel at the selected sites was easily achieved in all the eyes of the induced BRVO model; although catheterization was the most difficult maneuver because the force applied for inserting the polyimide tube was difficult to control, it must be sufficient to control placement of the tube without flattening or obstructing the tube or damaging tissue. At last, catheterization was accomplished only in 28 caprine eyes with no macroscopic damages to the tube, vessel wall, or retina, and with a sealed connection between the vessel and the polyimide tubing.

Combining vascular puncture, catheterization, and electrocoagulation, bypass of an occluded retinal vein segment was achieved in a total of 28 caprine eyes (Figure 1d). Three of the six failure cases were caused by severe neuroretinal edema, which occurred during prolonged surgery. Micro-vascular maneuvers could not continue in the overdelicate retina. Vascular laceration and retinal tears resulted from excessive force in carrying out catheterization in the other three failure cases.

\section{Evaluation of the patency of the retinal vascular bypass}

Patency of the vascular bypass was justified in a total of 23 caprine eyes (Figure 1e). In these caprine eyes, the submethyl-blue solution, which had been injected into the retinal vessel through the ophthalmic artery once again after retinal vein bypass surgery, flowed into the main vein vessel from the branch vessel through the vascular bypass, staining the main vein blue.

\section{Discussion}

The retinal vascular bypass surgery in our study with a combination of refine manipulations of vascular puncturing, catheterization, and endodiathermy has not been described in any literature as far as we know, and its level of difficulty is beyond the standard surgical maneuvers, such as stripping fibrovascular proliferation from proliferative diabetic retinopathy and internal limiting membrane from macular hole. In our previous study ${ }^{10}$ in the eyecup of arterially perfused caprine eye model, we demonstrated the feasibility of bypassing an occluded retinal main vessel segment between two openings with a polyimide tube in both the artery and the vein. In the present study, progress has been made. Vascular bypass of an occluded main vein segment with the polyimide tube was successfully performed through the small openings used in standard three-port PPV. The surgery was via a closed-sky approach rather than an open-sky approach, thus ensuring circumstances resembling that in in vivo condition.

A caprine eye model was selected for the microvascular surgery experiment because of its similar size to human retinal vessel as we described in our previous study, and hopefully the findings will be used as a reference for future experiments in the living eye.

The retinal blood circulation was simulated by arterial perfusion in the isolated caprine eye, and displacement of blood cleanly prevented thrombosis in the vessel and tube, which will most likely be confronted in in vivo surgery in the future study. Some key operative points during the establishment of retinal vascular perfusion included keeping air out of the perfusion system and keeping an appropriate flow rate, which have been described in our previous study. ${ }^{10}$

Keratoprosthesis has been widely used in the management of severe ocular trauma and proliferative retinopathy in the presence of keratopathy, which allow visualization of intraocular structures despite corneal opacities. ${ }^{12-14}$ The Landers wide-field TKP was introduced in this study to avoid cornea edema and opacity in in vitro eye, and it is designed with a convex anterior surface to provide $\times 2.29$ magnified retina image. There is no need of a optic fiber as keratoprosthesis provides a clear view of fundus directly with illumination of the operating microscope. Hence, during the surgery, keratoprosthesis provided a clear, stable, amplificatory, and stereoscopic real image of the retina. It also made the bimanual technique possible, having a key role of accomplishing retinal vascular bypassing.

Endodiathermy was a simple solution to establish the BRVO model and to connect the vessel and tube instead of vascular ligation, which was used in the eyecup model of our previous study, ${ }^{10}$ but was too difficult to be performed in the closed-sky eyeball model.

Endodiathermy should be performed with appropriate duration and power. In the study, a few eyes showed mild leakage of stain from the anastomose as a result of excessive endodiathermy.

In the closed-sky eyeball model, all of the maneuvers were performed through small openings used in standard three-port PPV. This created much of the difficulty of retinal vascular bypassing, leading to low success rate of the surgery. The unsuccessful cases were 
mainly caused by rupture of vascular, retinal breaks and retinal detachments. Physiological tremor during retinal microsurgery, reportedly $108 \mu \mathrm{m}$ in amplitude, ${ }^{15}$ was generally greater than the target blood vessel diameter, which was only $\sim 80 \mu \mathrm{m}$. Manual dexterity, precision, and perception were particularly important during surgery. Skilled operations were demanded to accomplish all of the maneuvers. From experiences we gained in our prior study ${ }^{10}$ and repeated trainings, a series of micro-vascular maneuvers with a good result were accomplished in 23 caprine eyes in the later period of this study.

We anticipated that the findings of this in vitro study would be useful for future experiments as the conditions of all the retinal micro-vascular manipulations performed in closed-sky eyeball were similar to that in in vivo eye. During retinal vascular procedures in the living eye, treatment of hemorrhages of vessels and thrombosis in the vessel-tube anastomotic stoma represents a serious challenge. The long-term patency of the polyimide tube used as a vessel bypass must be taken into consideration regarding the possibility of obstruction of the tube by fibrin in in vivo conditions. The intraoperative fluorescein angiography technique can be used to evaluate the patency of the retinal vascular bypass. ${ }^{16}$ Improved methods, instead of diathermy, should be explored to link the vessels and tubes in the future.

In conclusion, in the isolated arterially perfused caprine eye model of a closed-sky eyeball, we demonstrated the feasibility of bypassing an occluded retinal main vessel segment, and successfully justified the patency of retinal vessel bypass. These findings may be used as a reference for future experiments in the living eye.

\section{Summary}

\section{What was known before}

- As a very common retinal vascular disorder, branch retinal vein occlusion is short of effective therapies at present. Bypassing the occluded retinal vessel segment with artificial graft, which focused on the re-establishment of retinal venous outflow, has been demonstrated feasible in an animal eyecup model via an open-sky approach.

\section{What this study adds}

- In this study, vascular bypass surgery was successfully performed through the small openings used in standard three-port pars plana vitrectomy via a closed-sky approach rather than an open-sky approach, thus ensuring circumstances resembling that in in vivo condition. These findings may be used as a reference for future experiments in the living eye.

\section{Conflict of interest}

The authors declare no conflict of interest.

\section{Acknowledgements}

This work was supported by Grant 2006C23036 from the Bureau of Science and Technology of Zhejiang Province, China.

\section{References}

1 Branch Vein Occlusion Study Group. Argon laser photocoagulation for macular edema in branch vein occlusion. Am I Ophthalmol 1984; 98: 271-282.

2 Tameesh MK, Lakhanpal RR, Fujii GY, Javaheri M, Shelley $\mathrm{TH}, \mathrm{D}^{\prime}$ Anna S et al. Retinal vein cannulation with prolonged infusion of tissue plasminogen activator (t-PA) for the treatment of experimental retinal vein occlusion in dogs. Am J Ophthalmol 2004; 138: 829-839.

3 Osterloh MD, Charles S. Surgical decompression of branch retinal vein occlusions. Arch Ophthalmol 1988; 106: 1469-1471.

4 Garcia-Arumi J, Martinez-Castillo V, Boixadera A, Blasco H, Corcostegui B. Management of macular oedema in branch retinal vein occlusion with sheathotomy and recombinant tissue plasminogen activator. Retina 2004; 24: 530-540.

5 Horio N, Horiguchi M. Effect of arteriovenous sheathotomy on retinal blood flow and macular edema in patients with branch retinal vein occlusion. Am J Ophthalmol 2005; 139: 739-740.

6 Avci R, Inan U, Kaderli B. Evaluation of arteriovenous crossing sheathotomy for decompression of branch retinal vein occlusion. Eye 2008; 22: 120-127.

7 McAllister IL, Douglas JP, Constable IJ, Yu DY. Laser-induced chorioretinal venous anastomosis for nonischemic central retinal vein occlusion:evaluation of the complications and their risk factors. Am J Ophthalmol 1998; 126: 219-229.

8 Peyman GA, Kishore K, Conway MD. Surgical chorioretinal venous anastomosis for iachemic central retinal vein occlusion. Ophthalmic Surg Lasers 1999; 30: 605-614.

9 Weiss J. Retinal surgery for treatment of central vein occlusion. Ophthalmic Surg Lasers 2000; 31: 162-165.

10 Li-Jun Shen, Yi-Qi Chen, Li-Li Wei, Wei Wu, Zhao-Yang Wang, Yang Liu et al. Bypassing occluded retinal main vessel segments in isolated arterially perfused caprine eyes. Curr Eye Res 2009; 34: 415-420.

11 Schmidt JC, Chofflet J, Hörle S, Mennel S, Meyer CH. Three simple approaches to visualize the transparent vitreous cortex during vitreoretinal surgery. Dev Ophthalmol 2008; 42: 35-42.

12 Garcia-Valenzuela E, Blair NP, Shapiro MJ, Gieser JP, Resnick KI, Solomon MJ et al. Outcome of vitreoretinal surgery and penetrating keratoplasty using temporary keratoprosthesis. Retina 1999; 19(5): 424-429.

13 Gelender H, Vaiser A, Snyder WB, Fuller DG, Hutton WL. Temporary keratoprosthesis for combined penetrating keratoplasty, pars plana vitrectomy, and repair of retinal detachment. Ophthalmology 1988; 95: 897-901.

14 Gross JG, Feldman S, Freeman WR. Combined penetrating keratoplasty and vitreoretinal surgery with the Eckardt temporary keratoprosthesis. Ophthalmic Surg 1990; 21: 67-71.

15 Singh S, Riviere C. Physiological tremor amplitude during retinal microsurgery. Proceedings of the IEEE 28th Annual Northeast Bioengineering Conference; Philadelphia, PA, USA. IEEE (Institute of Electrical and Electronics Engineers): Philadelphia, PA, 2002, pp 171-172.

16 Horio N, Horiguchi M. Retinal blood flow analysis using intraoperative video fluorescein angiography combined with optical fiber-free intravitreal surgery system. Am J Ophthalmol 2004; 138: 1082-1083. 